\title{
A new modulation method for multilevel converters
}

\author{
Francisco Gordillo \\ Escuela Técnica Superior de Ingeniería, Universidad de Sevilla \\ Camino de los Descubrimientos s/n. \\ 41092 Sevilla, Spain \\ Phone: +34 954487345 \\ Fax: +34954487340 \\ Email: gordillo@us.es
}

\section{Acknowledgments}

The author would like to thank Profs. Juan M. Carrasco and E. Galván for their valuable comments and suggestions during fruitful discussions. This work has been funded under grant MINECO-FEDER DPI2013-41891-R.

\begin{abstract}
This paper presents a simple method for modulation in multilevel converters, which is hardly affected with the number of levels. The method is based on a simple graph, whose $x$-axis is directly related to the extra degree of freedom associated with component $\gamma$ (in the $\alpha \beta \gamma$ reference frame) of the desired reference vector. In the $y$-axis, the different possibilities for the phase averaged references are plotted. In this way, the degree of freedom is more explicit than in Space Vector Modulation (SVM) and it can be the basis of new algorithms to pursue additional control objectives such as voltage balancing or commonmode reduction, among others. A comparison with SVM based on examples is included, showing the benefits of the proposed modulation strategy.
\end{abstract}

\section{Keywords}

Multilevel converters, Modulation Strategies, Space Vector Modulation.

\section{Introduction}

In the control of power converters it is usual to work with averaged models where the discrete values of the gating signals are considered as continuous signals. In order to implement the control laws obtained with such models, a discretization stage, usually called modulation for extension of the well-known Carrier-Based Pulse Width Modulation (CB-PWM) method, needs to be implemented [1]. The objective of the modulation stage is the computation (and implementation) of the discrete values for the gating signals as a function of the computed averaged value that they should achieve. With the appearance of multilevel converters the number of modulation methods and their variants have growth considerably [2-4]. Among the modulations techniques, the Space Vector Modulation (SVM) plays important roles. Throughout its important benefits there is the explicit identification of redundancies [1], that is, different combinations of gating states that correspond to the same space vector. This redundancy, associated with component $\gamma$ (in the $\alpha \beta \gamma$ reference frame) of the reference voltage vector, can be used for different control objectives such as modulation index extension, capacitor voltage balancing, switching frequency reduction, fault tolerance or common-mode voltage elimination [3,5]. Nevertheless, as the number of level increases in multilevel converters, extensions of SVM increase their complexity. Different variants have been proposed in order to achieve these new control objectives modifying the 
original SVM algorithm [6-10]. Some variants even consider points different from the three nearest vectors $[11,12]$.

Space vector modulation can be divided in two stages [13]. In the first stage, the active vectors and their corresponding times are computed; in the second stage the sequence of the active vectors within the sampling period is chosen. This paper deals with the first of these two stages and a new procedure for choosing, in multilevel converters, the desired levels and their corresponding times for each phase is presented. The main benefits of the proposed method are, on the one hand, its simplicity that it is hardly affected by the number of levels; on the other hand, the degree of freedom associated with component $\gamma$ of the reference vector is much more explicit than in SVM. In fact, the method is based on a particular graph, presented in this paper, in which the $x$-axis is directly related to this component. Furthermore, the graph (and, consequently, the algorithm) changes just slightly when the number of levels increases. The new method is based on independent formulae for each phase and, thus, it could be considered a scalar modulation technique. Nevertheless, the vectorial nature of the modulation is taken into account since the formulae for the three phases are jointly considered in the graph previously mentioned. In this way, the redundancy of different combinations (e.g. ppp,ooo and $n n n$ in the three-level case) is also considered.

This paper does not present a new control method that improves the performance of previous techniques for particular converters but, instead, a new paradigm that may give rise to new control methods is introduced. In [14] this paradigm is used to obtain a new control procedure for voltage balance in 3phase, 5-level DCC rectifiers with minimization of number of switches.

The next section is devoted to the description of the method, while in Section III, the advantages and drawbacks with respect to SVM are discussed. In this section, some problems of SVM (such as a deceitful degree of freedom) are shown up. The paper ends with a section of conclusions.

\section{Description of the method}

The objective of the modulation stage is the computation and implementation of the duty cycles for each phase and level, in each sampling instant. The input data are the reference voltage $\alpha-\beta$ coordinates. In this paper, normalized voltages are considered in $a b c$ coordinates (denoted as $u_{a}, u_{b}$ and $u_{c}$ ) in such a way that, in the three-level case, $u_{a}=-1$ corresponds to the lower level for phase a, $u_{a}=0$ to the central level and $u_{a}=1$ to the upper level and analogously for the other phases. Similar normalizations can be defined for cases with higher number of levels. Normalization in $\alpha \beta \gamma$ coordinates must be consistent to the Clarke transformation giving rise to $u_{\alpha}, u_{\beta}$ and $u_{\gamma}$. Usually, at each sampling time, the desired (averaged) values of $u_{\alpha}$ and $u_{\beta}$ are computed by an outer controller that it is in charge of regulating powers and currents. The modulation stage converts these values to discrete states in $a b c$ coordinates. The modulation ends with a sequencing phase and the implementation of the gating signals, which are not the object of this paper.

As it is well known, a degree of freedom appears when choosing the discrete states: coordinate $u_{\gamma}$ is usually not given by the outer controller. This fact appears in SVM as redundant state vectors, that is, vectors in the $\alpha-\beta$ plane that correspond to several vectors in the $a b c$ space. The method proposed in this paper consideres explicitly this degree of freedom. For this, consider the inverse Clarke transformation from $\left(u_{\alpha}, u_{\beta}, u_{\gamma}\right)$ to $\left(u_{a}, u_{b}, u_{c}\right)$

$$
\left[\begin{array}{l}
u_{a} \\
u_{b} \\
u_{c}
\end{array}\right]=\sqrt{\frac{2}{3}}\left[\begin{array}{rrr}
1 & 0 & \frac{\sqrt{2}}{2} \\
-\frac{1}{2} & \frac{\sqrt{3}}{2} & \frac{\sqrt{2}}{2} \\
-\frac{1}{2} & -\frac{\sqrt{3}}{2} & \frac{\sqrt{2}}{2}
\end{array}\right]\left[\begin{array}{l}
u_{\alpha} \\
u_{\beta} \\
u_{\gamma}
\end{array}\right] .
$$

If $u_{\alpha}$ and $u_{\beta}$ are given, Eq. (1) can be interpreted as a vectorial function that gives $\left(u_{a}, u_{b}, u_{c}\right)$ for each 
value of $u_{\gamma}$. In order to simplify this equation, consider the following definitions

$$
\begin{aligned}
x & \stackrel{\text { def }}{=} \frac{1}{\sqrt{3}} u_{\gamma} \\
\eta_{a} & \stackrel{\text { def }}{=} \sqrt{\frac{2}{3}} u_{\alpha} \\
\eta_{b} & \stackrel{\text { def }}{=}-\frac{1}{\sqrt{6}} u_{\alpha}+\frac{1}{\sqrt{2}} u_{\beta} \\
\eta_{c} & \stackrel{\text { def }}{=}-\frac{1}{\sqrt{6}} u_{\alpha}-\frac{1}{\sqrt{2}} u_{\beta},
\end{aligned}
$$

Notice that $x$ depends on $u_{\gamma}$ while $\eta_{a}, \eta_{b}$ and $\eta_{c}$ are constants when $u_{\alpha}$ and $u_{\beta}$ are given. With this definition, Eq. (1) yields

$$
\begin{aligned}
& u_{a}(x)=\eta_{a}+x \\
& u_{b}(x)=\eta_{b}+x \\
& u_{c}(x)=\eta_{c}+x .
\end{aligned}
$$

These equations correspond to the well-known fact that the homopolar component provides an associated degree of freedom [15-17]. Equations (6)-(8) correspond to three parallel lines as represented in Fig. 1 for $u_{\alpha}=1$ and $u_{\beta}=0.5$. In this figure the horizontal lines at $u_{a b c}=\{-1,0,1\}$ represent the three discrete implementable states for the three-level case. Notice that the extension to higher number of levels would lead to minor changes: only some horizontal lines should be added.

The vertical lines in Fig. 1 show the limits of implementable cases. Notice that, in this figure, for the values of $x$ inside the vertical lines, the corresponding value for $u_{a}, u_{b}$ and $u_{c}$ are all of them inside the implementable interval $[-1,1]$ (in three-level converters). The values of $x$ for the vertical lines can be defined in the following way: $x_{\min }$ is the maximum of the three values of $x$ such that one of the three lines takes the minimum level, and $x_{\max }$ is the minimum of the values of $x$ such that one of the three lines takes the maximum level. The condition for the feasibility of the proposed method is that $x_{\min } \leq x_{\max }$. In the next section, it will be shown that there is not extra conservativeness in this condition. When the input values $u_{\alpha}$ and $u_{\beta}$ change so do the sloping lines as well as the vertical limits.

The modulation procedure can be divided in two steps. The first one is based on Eqs. (6)-(8) as well as their associated graph, Fig. 1. The second step is just scalar modulation.

Step 1 Selection of the value of $x$ and computation of $u_{a}, u_{b}$ and $u_{c}$. In this step a value for $x$ in the feasible interval should be chosen. The associated degree of freedom can be used for additional control objectives. As it has been said, the input data $u_{\alpha}-u_{\beta}$ are set by an outer controller in order to regulate the currents and powers, but other control objectives appear in multilevel converters such as the voltage balance in NPC converters. It is well known that $u_{\gamma}$ can be used for this purpose. One advantage of the proposed method is to clarify and put explicitly this degree of freedom as will be discussed in the next section. The specific selection methods are to be defined and will depend on the control objective and on the number of levels. This paper is restricted to present a new paradigm that may give rise to new control methods.

One possibility is to choose a value of $x$ such that the level corresponding to one of the phases does not suffer any switch in order to diminish the number of commutations. This criterium can also be accomplished in some combinations of the Nearest Three Vectors SVM (NTV-SVM) [6]. In order to achieve this situation, $x$ has to be chosen in the (feasible) intersection of any of the horizontal lines in Fig. 1 with the sloping lines. In the example corresponding to this figure, there are three of these 


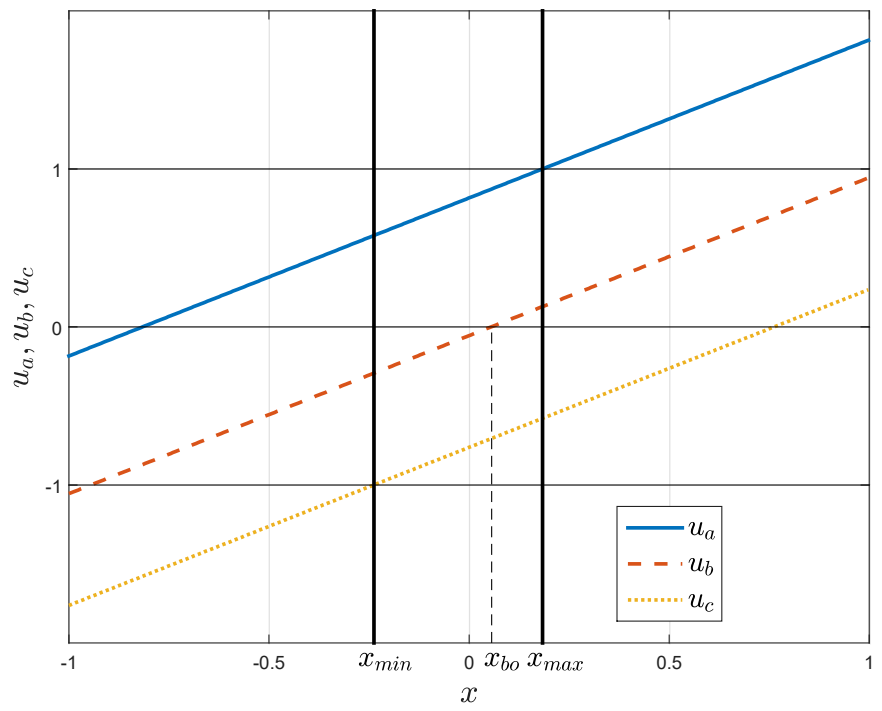

Fig. 1: Achievable phase levels for $u_{\alpha}=1$ and $u_{\beta}=0.5$ in 3-level converters. 
intersections, but for other values of $u_{\alpha}$ and $u_{\beta}$ the number of intersections may vary. Of course, these intersections are easy to be computed analytically. Different criteria may be used for the choice of $x$ in order to achieve other control objectives.

Once the value of $x$ has been selected, the corresponding values for $u_{a}, u_{b}$ and $u_{c}$ are computed using (6)-(8).

Step 2 The second step is the discretization of the continuous values $u_{a}, u_{b}$ and $u_{c}$. As they are treated as three scalar values, this step is straightforward. The obvious procedure would be a standard scalar modulation, that is, at first, the computation of the two adjacent levels $l_{i}^{1}$ and $l_{i}^{2}$ and, secondly, their respective duty cycles:

$$
\begin{aligned}
l_{i}^{1} & =\text { floor }\left(u_{i}\right) \\
l_{i}^{2} & =l_{i}^{1}+1 \\
d\left(l_{i}^{2}\right) & =u_{i}-l_{i}^{1} \\
d\left(l_{i}^{1}\right) & =1-d\left(l_{i}^{2}\right),
\end{aligned}
$$

for $i=a, b, c$

Notice that if component $\gamma$ of the reference voltage is given, Step 1 should be omitted and Step 2 leads to a (trivial) alternative to 3D-SVM [7].

Notice also that another degree of freedom may appear in this step. Some existing modulation strategies do not avoid switching among more than two levels in each sampling time, in order to pursue other control objectives such as the voltage balance among capacitors in NPC converters [18, 19]. Using this idea, Step 2 can be modified and different algorithms for discretization can be used.

Example As an example of application, consider that, in a concrete sampling instant, the values of $u_{\alpha}$ and $u_{\beta}$ correspond to the case of Fig. $1\left(u_{\alpha}=1\right.$ and $\left.u_{\beta}=0.5\right)$. In this case definitions (3)-(5) give $\eta_{a}=0.8165, \eta_{b}=-0.0547$ and $\eta_{c}=-0.7618$. Since $\eta_{a}$ is the maximum of these values, $x_{\max }=1-\eta_{a}=$ 0.1835 . On the other hand, as the minimum $\eta$ is $\eta_{c}, x_{\min }=-1-\eta_{c}=-0.2382$.

Suppose that, in the considered sampling instant, it is desired to avoid commutations in one of the phases, say phase $b$. It suffices to find a value of $x$ such that the associated value of $u_{b}$ is $-1,0$ or 1 . It can be checked in Fig. 1 that the line corresponding to phase $u_{b}$ reaches the level $u_{b}=0$ for a feasible value of $x$ (marked as $x_{b o}$ in the figure), $x_{\min } \leq x_{b o} \leq x_{\max }$. This computation can be performed in an algorithmic way, since using (7) for $u_{b}=0$, it is obvious that $x_{b o}=-\eta_{b}=0.0547$. Substitution of this value in (6) and (8) yields $u_{a}=0.8712$ and $u_{c}=-0.7071$. Therefore, phase $a$ will commute during the sampling period between levels 0 and 1, while phase $c$ will commute between 0 and -1 . Of course, phase $b$ will be connected to level 0 the full sampling period, since $u_{b}=0$.

As was said above, the procedure is valid for any number of levels. The only modification would be that more horizontal lines, one for each level, would be added to Fig. 1. Figure 2 shows an example of the graph for the five-level case. Equations (9-12) do not change with the number of levels.

\section{Comparison with Space Vector Modulation}

The main advantages of space vector modulation with respect to other scalar modulation techniques or with respect to CB-PWM are well known. Some of them are the following:

1. The degree of freedom related to component $\gamma$ of the reference voltage is explicitly identified. This degree of freedom is shown in the redundant states: space vector points that can be implemented using different combinations of switches. For example, in two level converters the hexagon central point can be implemented by the combinations $p p p$ and $n n n$. 


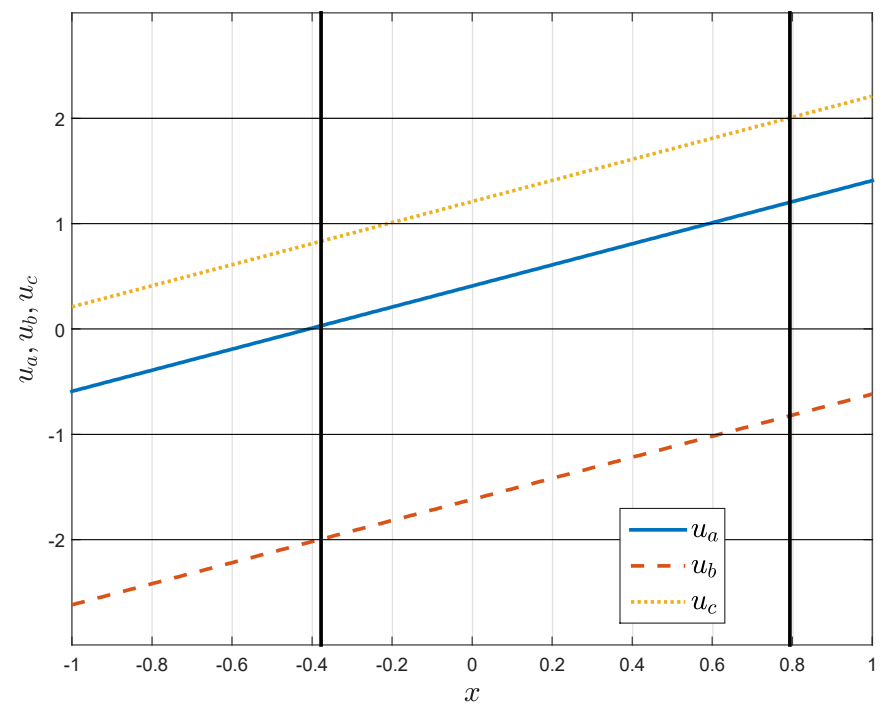

Fig. 2: Achievable phase levels for $u_{\alpha}=0.5$ and $u_{\beta}=-2$ in five-level converters. 
2. This degree of freedom can be used for the search of sequences with low number of commutations. Indeed, if NTV-SVM strategy is used, there are combinations such that one phase does not present any switch while the other phases commute just once. Furthermore, the switches are between two consecutive levels, avoiding dangerous commutations between non-consecutive levels. Other control objectives, such e.g. voltage balance in NPC converters, may require the increase of commutations by choosing other commutations and, even other vectors (not just the nearest three) can be chosen [18].

3. Space vector can be used for obtaining overmodulation techniques that have been applied with success and are now industry standards.

4. The space vector graph may inspire algorithms that take into account other control objectives. See $[12,20]$ for examples of application to common mode reduction.

The objective of this section is to show that the proposed modulation strategy keeps the first two advantages and even it outperforms them. The possible direct use of the proposed method for overmodulation is not studied in this paper and it is left as future work, but in any case the modified SVM reference vector can be used as the vector input of the proposed method in order to work in the overmodulation region.

Degree of freedom One of the benefits of SVM with respect to CB-PWM [1] is the explicit identification of the additional degree of freedom related to the redundancy of some space vectors and to component $\gamma$ of the reference voltage. In the previous section, it has been seen that this degree of freedom is more explicit in the proposed method as the $\gamma$ component is the independent variable in Eqs. (6)-(8), and the horizontal axis in Fig. 1.

In the following it is shown by means of examples that the proposed modulation strategy can yield equivalent results to SVM and, thus, it preserves the degree of freedom.

Consider, as an example, that the normalized vector input is given by $u_{\alpha}=1, u_{\beta}=0.5$. This choice, that corresponds to the case of Fig. 1, has been arbitrarily chosen and does not correspond to any case specially suited for the proposed method. Similar reasonings can be done for other values. This space vector is shown in the Fig. 3 and the tree nearest points correspond to (the usual notation $p, o, n$ is used for levels $-1,0$ and 1 respectively)

- Point A: ppo or oon.

- Point B: pon

- Point C: poo or onn.

Some possible combinations of these nearest three points are:

1. ppo-poo-pon.

2. pon-oon-onn.

3. poo-pon-oon-onn.

4. ppo-poo-pon-onn-oon.

These four combinations, which are not the only ones when using the NTV-SVM, represent different cases. Combinations 1 and 2 only use three vectors (i.e. no redundant states are used) and they have been chosen in order to minimize the number of commutations: in each one, one phase does not commute and the other two phases suffer just one switch (of course, in the sequencing stage, a state can be divided in two intervals giving rise to more commutations, but remember that this paper and this discussion are 


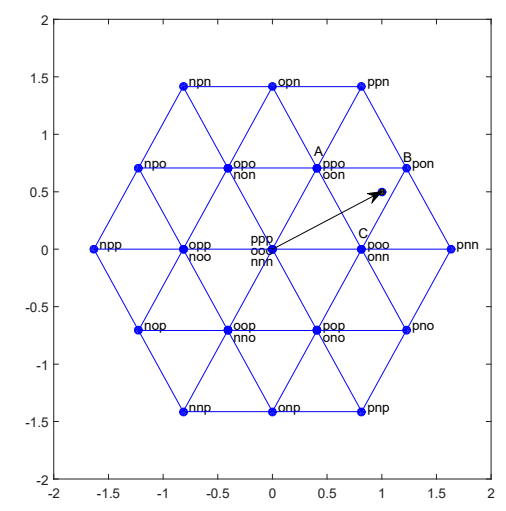

Fig. 3: Space vector for $u_{\alpha}=1$ and $u_{\beta}=0.5$.

devoted to the modulation stage; the sequencing stage will not change the conclusions of this discussion). Combinations 3 and 4 use redundant states, still using the nearest three points. These combinations yield a higher number of commutations, but they can be used for other control purposes. Notice that in combination 4 phase $b$ commutes among the three levels. Once a combination has been chosen, the duty cycles can be computed, but they are not necessary in this discussion.

Let us see that this four combinations are among the infinite number of combinations that can be generated using the proposed modulation technique. Notice that in combination 1, phase $a$ is always at the positive level. The associated value of $x$ corresponds to intersection of the line associated with phase $a$ and $u_{a}=1$ (yielding in this case to $x_{\max }$, see Fig. 1). By means of visual inspection in Fig. 1 it can be checked that phase $b$ takes a value between 0 and 1 and therefore, the proposed algorithm for step 2 would result in this phase switching between $o$ and $p$. Phase $c$ takes a value between -1 and 0 and the Step-2 algorithm would result in commutations between $n$ and $o$. Using Eqs. (9)-(12) the numerical values for the duty cycles can be directly obtained. It is easy to see that the resultant commutation strategy is equivalent to combination 1.

A similar reasoning can be applied to combination 2. In this case, phase $c$ is the one that does not commute. It stays at level $n$ and corresponds in Fig. 1 to the vertical line where the $u_{c}$ line takes the value -1 (in this case it corresponds to $x=x_{\min }$ ). For this value of $x, u_{a}$ takes a value between 0 and 1 and $u_{b}$ between -1 and 0 . The trivial choice in Step 2 leads to combination 2.

There are infinite possible sets of duty cycles that yield combination 3 since there exists an additional degree of freedom, corresponding to different values of $u_{\gamma}$. This can be verified in Fig. 1. Denoting $x_{b o}$ as the value of $x$ such that $u_{b}=0$ it is direct to see that combination 3 can be obtained by any value of $x$ in the interval $\left(x_{\min }, x_{b o}\right)$.

Finally, combination 4 presents another additional degree of freedom since phase $b$ commutes among the three levels. It can be obtaining for any values of $x$ in the interval $\left(x_{\min }, x_{\max }\right)$ but introducing the 
new degree of freedom in step 2 for phase $b$ : the value of $u_{b}$ associated with the selected value of $x$ can be implemented using the three states by means of infinite combinations of weighting coefficients. Notice that these two types of degrees of freedom (the one associated with the value of $u_{\gamma}$, and the one associated with the commutation among three levels) appear separated in the two steps of the proposed method, but they are mixed in SVM.

False degree of freedom in SVM Continuing with the same example $\left(u_{\alpha}=1, u_{\beta}=0.5\right)$, consider combination 1. As it has been said above, this combination corresponds to $x=x_{\max }$. The associated values for the normalized averaged levels are $u_{a}=1, u_{b}=0.1289, u_{c}=-0.5782$. Figure 4 show two sequences that correspond to these duty cycles (this sequences have been arbitrarily chosen, without taken into account any other criteria such as low harmonic distortion). The first sequence corresponds to vectors $p p o-p o o-p o n$, which corresponds to combination 1, but the second sequence corresponds to $p p n-p o n-p o o$, which is a different one. Furthermore, the state ppn is not one of the nearest three vectors. The conclusion of this reasoning is that the space vector approach makes differences among combinations that are identical when they are implemented. The vectorial nature of the space vector approach considers some kind of "synchronization" among the phases. The advantages of such synchronization are not clear. In the proposed method, the two combinations of Fig. 4 are identical as they correspond to the same value of $x$, that is, the same value of $u_{\gamma}$. In fact, the differences between then are related to the sequencing phase and they should not correspond to the modulation or discretization stage.

Modulation limits and overmodulation As it was said in the previous section, the proposed modulation method only works when the two vertical lines of Fig. 1 are in the correct order, that is, $x_{\min } \leq x_{\max }$. In the following, it will be shown that this constraint is equivalent to the SVM requirement that the vector $\left(u_{\alpha}, u_{\beta}\right)$ lies inside the outer SVM hexagon. Indeed, the condition for the existence of implementable cases is $x_{\min } \leq x_{\max }$, that is

$$
\max _{i=a, b, c}\left(x: u_{i}(x)=l_{\min }\right) \leq \min _{i=a, b, c}\left(x: u_{i}(x)=l_{\max }\right),
$$

where $l_{\min }$ and $l_{\max }$ are the indexes associated with the minimum and maximum levels, respectively. Taking into account the fact that the three lines are parallel, the value of $i$ cannot be the same in both sides of this inequality. Therefore, the possible combinations of the min and max operators are six. Each of them yields a linear inequality. It is straightforward to check that these six linear inequalities give rise to the six sides of the SVM hexagon. Therefore, the range of validity of the proposed method is the same as the one of SVM.

Beyond the modulation limits, the overmodulation region exists where SVM provides several useful strategies [21] based on modifying the reference vector. The possible direct use of the proposed method is left as future work, but in any case the modified SVM reference vector can be used as the vector input of the proposed method in order to work in the overmodulation region. In this way, it is possible to combine the advantages of both methods, SVM and the one proposed in this paper.

Efficient use of the redundancy Another advantage of the proposed modulation technique is that it can use the SVM redundancy, that is, the degree of freedom associated with $u_{\gamma}$, without increasing the number of commutations. Notice that in SVM this degree of freedom is reflected in different vector states that correspond to the same space vector (see Fig. 3). In this figure, it can be seen that the three phases suffer commutations in the different states associated with any space vector. Any algorithm that takes advantage of this redundancy may lead to an increment in the number of switches. It could even yield one phase commuting among more than two levels. 

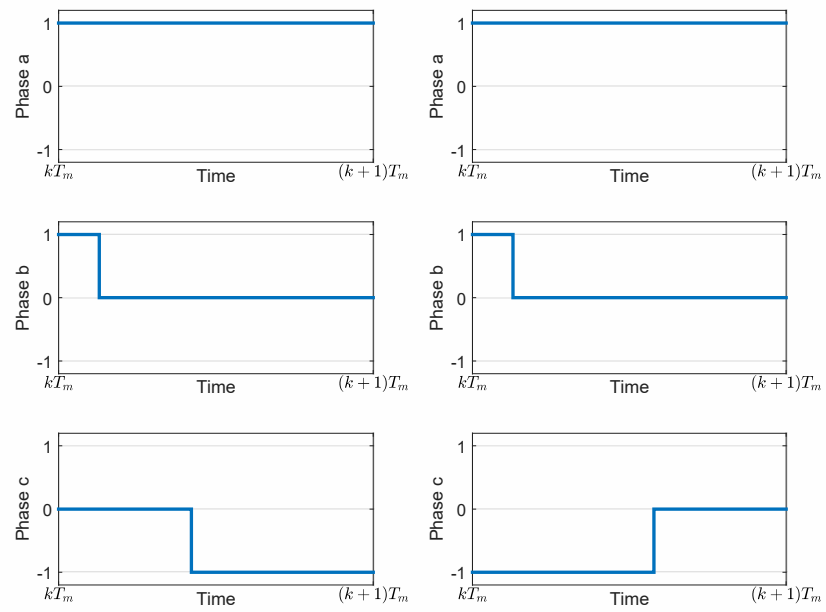

Fig. 4: Left and right: Two arbitrary sequences for $u_{a}=1, u_{b}=0.1289, u_{c}=-0.5782$. Sampling time is denoted by $T_{m}$. 
With the proposed method, it is possible to cover all the possibilities associated with the $u_{\gamma}$ degree of freedom (step 1) at the same time that the number of levels of each phase is at most two (step 2). The cases for which one phase can remain in one level are easily identifiable in step 1.

\section{Conclusion}

In this paper, a new modulation method has been proposed. The method is extremely simple and this is its main advantage with respect to Space Vector Modulation. It has been shown, by means of examples, that not only the proposed method incorporates the extra degree of freedom present in SVM, but this degree of freedom is more explicit in such a way that the different possibilities related to it are associated with the $x$-axis of a graph. Furthermore, it has been shown that SVM may propose as different, vectors that in fact are equivalent, giving rise to a deceptive degree of freedom.

Perhaps the most important advantage of the proposed method is the very low increment of its complexity as the number of levels grows. The examples of this paper correspond to the three-level case but they can be directly extended to cases with higher number of levels.

\section{References}

[1] D. Grahame Holmes and Thomas A. Lipo. Pulse Width Modulation for Power Converters: Principles and Practice. IEEE Press, 2003.

[2] Leopoldo G. Franquelo, José Rodríguez, José I. León, Samir Kouro, Ramón Portillo, and María A. M. Prats. The age of multilevel converters arrives. IEEE Industrial Electronics Magazine, 2(2):28-39, jun 2008.

[3] J. Rodríguez, L.G. Franquelo, S. Kouro, J.I. Leon, R.C. Portillo, M.A.M. Prats, and M.A. Perez. Multilevel Converters: An Enabling Technology for High-Power Applications. Proceedings of the IEEE, 97(11):1786-1817, nov 2009.

[4] S. Kouro, M. Malinowski, K. Gopakumar, J. Pou, L. G. Franquelo, J. Rodriguez, M. A. Perez, and J. I. Leon. Recent Advances and Industrial Applications of Multilevel Converters. IEEE Transactions on Industrial Electronics, 57(8):2553-2580, aug 2010.

[5] O.L. Sánchez. Space Vector Pulse-Width Modulation for Multilevel Multiphase Voltage-Source Converters. PhD thesis, Universidad de Vigo, 2008.

[6] N. Celanovic and D. Boroyevich. A fast space-vector modulation algorithm for multilevel threephase converters. IEEE Transactions on Industry Applications, 37(2):637-641, 2001.

[7] M.M. Prats, L.G. Franquelo, R. Portillo, J.I. Leon, E. Galvan, and J.M. Carrasco. A 3-D space vector modulation generalized algorithm for multilevel converters. IEEE Power Electronics Letters, 99(4):110-114, dec 2003.

[8] Sergio Busquets Monge, Sergio Somavilla, Josep Bordonau, and Dushan Boroyevich. Capacitor Voltage Balance for the Neutral-Point- Clamped Converter using the Virtual Space Vector Concept With Optimized Spectral Performance. IEEE Transactions on Power Electronics, 22(4):1128-1135, jul 2007.

[9] S. Jon Finney, A. Massoud, and B.W. Williams. An SVM Algorithm to Balance the Capacitor Voltages of the Three-Level NPC Active Power Filter. IEEE Transactions on Power Electronics, 23(6):2694-2702, nov 2008.

[10] M. Saeedifard, R. Iravani, and J. Pou. Control and DC-capacitor voltage balancing of a space vector-modulated five-level STATCOM. IET Power Electronics, 2(3):203-215, may 2009. 
[11] H.A. Hotait, A.M. Massoud, S.J. Finney, and B.W. Williams. Capacitor voltage balancing using redundant states of space vector modulation for five-level diode clamped inverters. IET Power Electronics, 3(2):292, mar 2010.

[12] Nho-Van Nguyen, Tam-Khanh Tu Nguyen, and Hong-Hee Lee. A Reduced Switching Loss PWM Strategy to Eliminate Common-Mode Voltage in Multilevel Inverters. IEEE Transactions on Power Electronics, 30(10):5425-5438, oct 2015.

[13] Dorin O Neacsu. Space Vector Modulation An Introduction. In IECON 2001 - 27th Annual Conference of the IEEE Industrial Electronics Society, pages 1583-1592, 2001.

[14] Francisco Gordillo, Fabio Gómez-Estern, and Francisco Salas. An optimization approach for modulation in multilevel converters. Submitted to the 42nd Annual Conference of the IEEE. 2016.

[15] C.B. Jacobina, A.M. Nogueira Lima, E.R.C. da Silva, R.N.C. Alves, and P.F. Seixas. Digital scalar pulse-width modulation: a simple approach to introduce nonsinusoidal modulating waveforms. IEEE Transactions on Power Electronics, 16(3):351-359, may 2001.

[16] Arnaud Videt, Philippe Le Moigne, Nadir Idir, Philippe Baudesson, and Xavier Cimetiere. A New Carrier-Based PWM Providing Common-Mode-Current Reduction and DC-Bus Balancing for Three-Level Inverters. IEEE Transactions on Industrial Electronics, 54(6):3001-3011, dec 2007.

[17] Iraide Lopez, Salvador Ceballos, Josep Pou, Jordi Zaragoza, Jon Andreu, Inigo Kortabarria, and Vassilios G. Agelidis. Modulation Strategy for Multiphase Neutral-Point-Clamped Converters. IEEE Transactions on Power Electronics, 31(2):928-941, feb 2016.

[18] S. Busquets-Monge, J. Bordonau, D. Boroyevich, and S. Somavilla. The nearest three virtual space vector PWM - a modulation for the comprehensive neutral-point balancing in the three-level NPC inverter. IEEE Power Electronics Letters, 2(1):11-15, mar 2004.

[19] Francisco Umbría, Francisco Gordillo, and Francisco Salas. Model-Based NPC Converter Regulation for Synchronous Rectifier Applications. In IECON 2014 - 40th Annual Conference of the IEEE Industrial Electronics Society, pages 4669-4675. IEEE, oct 2014.

[20] J. Rodríguez, J. Pontt, P. Correa, P. Cortes, and C. Silva. A New Modulation Method to Reduce Common-Mode Voltages in Multilevel Inverters. IEEE Transactions on Industrial Electronics, 51(4):834-839, aug 2004.

[21] Keliang Zhou and Danwei Wang. Relationship between space-vector modulation and three-phase carrier-based PWM: a comprehensive analysis. IEEE Transactions on Industrial Electronics, 49(1):186-196, 2002. 